\title{
Can one detect small-scale turbulence from standard meteorological radiosondes?
}

\author{
R. Wilson ${ }^{1}$, F. Dalaudier ${ }^{1}$, and H. Luce ${ }^{2}$ \\ ${ }^{1}$ UPMC Univ Paris 06, Univ. Versailles St-Quentin, UMR8190, CNRS/INSU - LATMOS-IPSL, 78280, Guyancourt, France \\ ${ }^{2}$ Univ. du Sud Toulon-Var, UMR6017, CNRS/INSU - LSEET, 83957, La Garde Cedex, France
}

Received: 23 November 2010 - Published in Atmos. Meas. Tech. Discuss.: 10 February 2011

Revised: 13 April 2011 - Accepted: 21 April 2011 - Published: 4 May 2011

\begin{abstract}
It has been recently proposed by Clayson and Kantha (2008) to evaluate the climatology of atmospheric turbulence through the detection of overturns in the free atmosphere by applying a Thorpe analysis on relatively low vertical resolution (LR) profiles collected from standard radiosoundings. Since then, several studies based on this idea have been published. However, the impact of instrumental noise on the detection of turbulent layers was completely ignored in these works. The present study aims to evaluate the feasibility of overturns detection from radiosoundings. For this purpose, we analyzed data of two field campaigns during which high-resolution (HR) soundings $(10-20 \mathrm{~cm})$ were performed simultaneously with standard LR soundings. We used the raw data of standard meteorological radiosondes, the vertical resolution ranging from 5 to $9 \mathrm{~m}$.

A Thorpe analysis was applied to both LR and HR potential temperature profiles. A denoising procedure was first applied in order to reduce the probability of occurrence of artificial overturns, i.e. potential temperature inversions due to instrumental noise only. We then compared the empirical probability density functions (pdf) of the sizes of the selected overturns from LR and HR profiles.

From HR profiles measured in the troposphere, the sizes of the detected overturns range from 4 to $\sim 1000 \mathrm{~m}$. The shape of the size pdf of overturns is found to sharply decrease with increasing scales. From LR profiles, the smallest size of detected overturns is $\sim 32 \mathrm{~m}$, a similar decrease in the shape of the pdf of sizes being observed. These results suggest
\end{abstract}

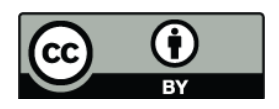

Correspondence to: R. Wilson (richard.wilson@upmc.fr) that overturns, resulting either from small-scale turbulence or from instabilities, can indeed be detected from meteorological radiosonde measurements in the troposphere and in the stratosphere as well. However they are rather rare as they belong to the tail of the size distribution of overturns: they only represent the $7 \%$ largest events in the troposphere, and $4 \%$ in the stratosphere.

\section{Introduction}

Thorpe (1977) proposed an elegant and simple-to-use method allowing to identify and to characterize overturns produced by turbulent patches from in situ measurements in oceans and lakes. The method is based on the comparison between an observed vertical profile of potential density and a reference profile corresponding to a minimum state of available potential energy. The reference profile is built by sorting in increasing order the observed potential density profile. It corresponds to an adiabatic re-arrangement of the observed fluid parcels. This method can be applied to atmospheric data as well by considering potential temperature for non saturated air instead of potential density. Since a vertical profile of potential temperature in a stably stratified atmosphere is a monotonic function of altitude, overturns can be easily identified by comparing the observed and sorted profiles. Such overturns are the signature of convectively unstable regions which can be due either to convective or dynamical instabilities of the flow (prior to the onset of developed turbulence) or to inversions produced by turbulent eddies. The convective and dynamical instabilities giving rise to overturns are expected to break down and to produce small-scale

Published by Copernicus Publications on behalf of the European Geosciences Union. 
turbulence. The detected overturns are thus believed to be associated with the occurrence of turbulent events. However, we cannot distinguish from the only in situ measurements, overturns resulting from the rolling up of air due to instabilities before turbulent breakdown from those produced by turbulent eddies in a mixing layer.

Simultaneous observations of convectively unstable regions from in situ and additional instruments (such as radars, lidars or aircrafts, ...) remain very sparse in the free atmosphere. Luce et al. (2002) reported observations of strong and isotropic radar echoes (suggesting the presence of turbulence) probably related to several unstable or nearly neutralized layers which were detected by high-resolution balloon measurements. These observations support the hypothesis of turbulent events related to overturns in the potential temperature profile. However, such a comparison cannot be fully conclusive since the air masses encountered by the balloon passed at distances of few kilometers from the radar, 1.5 to $16 \mathrm{~km}$, with a time delay of about 10-16 min. Further experimental validation is still desirable.

In principle, the Thorpe method can be applied to standard sounding data, either conductivity-temperaturedensity (CTD) in the oceans or lakes, (e.g. Thorpe, 1977; Galbraith and Kelley, 1996; Ferron et al., 1998; Alford and Pinkel, 2000) or pressure-temperature (PT) in the atmosphere (Luce et al., 2002; Gavrilov et al., 2005; Clayson and Kantha, 2008; Wilson et al., 2010). It has been recently proposed by Clayson and Kantha (2008) to apply Thorpe analyses to the huge data-base of meteorological radiosondes (hereafter noted RS), in order to infer the space-time variability of atmospheric turbulence in the free atmosphere. Since then, several such studies were published (Alappattu and Kunhikrishnan, 2010; Nath et al., 2010). Also, Balsley et al. (2010) proposed to use slow-ascent radiosondes for observing turbulence in the atmosphere.

However, the issue of instrumental noise was not addressed at all in these aforementioned works. From our point of view, the issue of noise is a key point of the Thorpe method, that must be considered with great care. Oceanographers long-time know that instrumental noise can generate artificial overturns which are very difficult to distinguish from real ones (e.g. Thorpe, 1977; Galbraith and Kelley, 1996; Ferron et al., 1998; Gargett and Garner, 2008). Surprisingly, except a few works (Gavrilov et al., 2005; Wilson et al., 2010), the issue of noise is generally ignored in most studies dealing with atmospheric turbulence arguing that: "it is less of an issue in the free atmosphere, where the mean overall stratification is quite strong..." (Clayson and Kantha, 2008). From our point of view, as shown by Wilson et al. (2010), this assertion does not hold, neither for standard meteorological measurements, nor for HR atmospheric measurements, because nearly neutral layers are very common, even at the vertical resolution of the standard radiosondes.
Hereafter, according to the terminology used by Johnson and Garrett (2004), an inversion is defined as a localized decrease of potential temperature versus height, whatever its origin may be, either induced by turbulent motions or by noise. (The present definition should not be confused with the standard definition of an inversion in meteorology which refers to a layer for which temperature increases whith height, for instance at the top of the planetary boundary layer.) The term overturn will specifically refer to an inversion resulting from atmospheric motions (developed turbulence or convective overturns produced by instabilities in the flow). The selection of artificial inversions as overturns, may result in a dramatic overestimation of their frequency of occurrence, thus leading to overestimates of both the turbulent fraction of the atmosphere and the turbulence energy. The space-time inhomogeneity of turbulence can give rise to diffusion coefficient estimates ranging over several orders of magnitude Wilson (2004). It is therefore crucial to apply a quantitative procedure allowing to discriminate overturns from noise-induced inversions.

A quantitative method for selecting overturns in a potential temperature profile has been recently proposed by Wilson et al. (2010). The method is based on a hypothesis test applied to the data range within the detected inversions. The range of a data sample is defined as the difference between the maximum and the minimum values in that sample (the term "sample" refers here to a subset of a dataset). The detected inversions which cannot be distinguished from artificial inversions, i.e. inversions induced by instrumental noise, are not selected as overturns. The selection method will be described in more details later.

The present paper addresses the following two questions:

1. Can we detect small scale turbulence (or more exactly overturns) from meteorological radiosondes in the troposphere and lower stratosphere?

2. If yes, what are the sizes of the detectable overturns considering the vertical resolution and the noise level of radiosonde measurements?

In order to address these issues, we analyzed the datasets of two field campaigns during which high-resolution (HR) balloon soundings, 10 or $20 \mathrm{~cm}$ vertical resolution, and standard RS with relatively low-resolution (LR), 5 to $9 \mathrm{~m}$ vertical resolution, were simultaneously performed: the SFT campaign (1998) and the MUTSI campaign (2000). (SFT stands for Structure Fine de Temperature) and MUTSI for MU radar, Temperature Sheets and Interferometry). Seven (fourteen) RS were launched during the SFT and MUTSI campaigns respectively. One of the seven LR flights of the SFT campaign is not used in this study as the ascent speed was abnormally slow.

The paper is structured as follows. The datasets and data processing methods are described in Sect. 2. The analysis methods of the potential temperature profiles are presented 

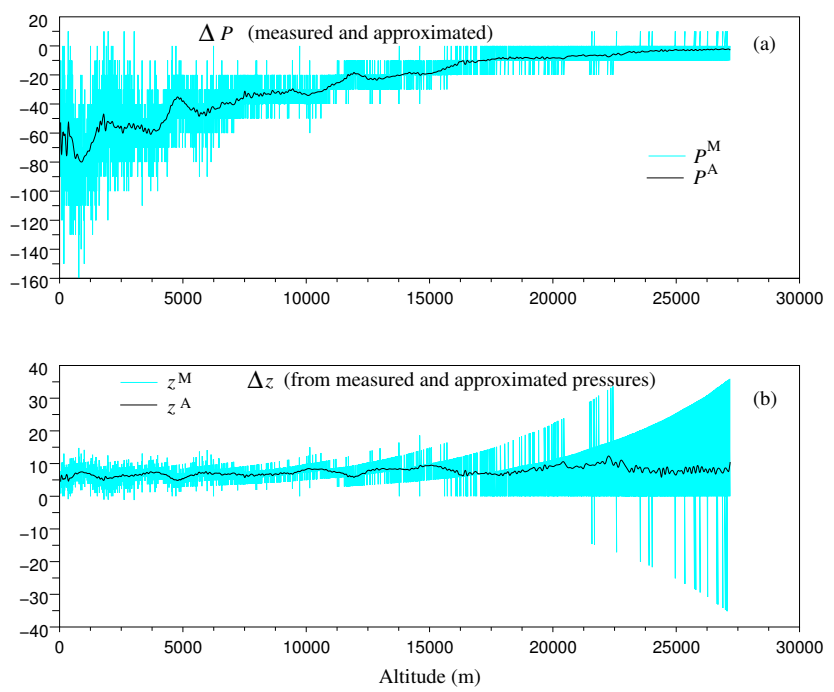

Fig. 1. (a) The first differences of measured pressure $\Delta P$ (cyan) and approximated pressures (black) for thr MUTSI-LR10 flight. (b) The first difference of altitudes $\Delta z$ inferred from raw measurements (cyan) and by using the approximated pressures (black).

in Sect. 3, a quantitative criterion for the selection of the overturns being described. In Sect. 4, we present the distribution in size of the selected overturns from both LR and HR profiles in the troposphere and in the lower stratosphere. Discussion and conclusions are given in the final section.

\section{Dataset and data processing}

\subsection{The datasets}

Meteorological RS provide temperature, pressure and relative humidity measurements. With the aim of detecting small scale motions, we used the raw RS data during the SFT and MUTSI campaigns. The RS data were obtained with Vaisala radiosondes RS80G (SFT) and RS90G (MUTSI) at an initial sampling frequency of $0.7 \mathrm{~Hz}$. The altitude resolution is variable due to the variations of the vertical ascent velocity of the balloon.

For each of the campaigns (SFT and MUTSI), three of the balloons carried both HR and LR sensors. The HR profiles were acquired with a sampling frequency of $25 \mathrm{~Hz}$ for the SFT flights and $50 \mathrm{~Hz}$ for the MUTSI flights. The reader can find more details about the HR measurements in Luce et al. (2002) and Gavrilov et al. (2005). The HR and LR sensors were on the same gondola, and the data were acquired simultaneously.

\subsection{Construction of potential temperature profiles from raw data of meteorological radiosondes}

For the RS80 and RS90 Vaisala radiosondes used in this study, the measured temperature and pressure are quantized with a resolution of $0.1 \mathrm{~K}$ and $10 \mathrm{~Pa}$ respectively. As previously mentioned, the measurements are irregularly sampled with altitude.

In order to perform a Thorpe analysis, it is necessary to have data sampled at a regular vertical step, the reference profile being a vertical rearrangement of the observed profile. The measured profiles need to be resampled at a regular vertical interval $\delta z$. The first task is to estimate the altitude of the measurements. By assuming hydrostatic equilibrium, the $i$-th altitude difference, $\Delta z_{i}^{\mathrm{M}}=z_{i+1}^{\mathrm{M}}-z_{i}^{\mathrm{M}}$, inferred from the measured pressure and temperature at level $i, P_{i}^{\mathrm{M}}$ and $T_{i}^{\mathrm{M}}$ respectively, is given by

$\Delta z_{i}^{\mathrm{M}}=-\frac{R T_{i}^{\mathrm{M}}}{g_{i}} \frac{\Delta P_{i}^{\mathrm{M}}}{P_{i}^{\mathrm{M}}}$

where $R$ is the specific gas constant for air $\left(\mathrm{J} \mathrm{kg}^{-1} \mathrm{~K}^{-1}\right)$ and $g_{i}$ the acceleration of gravity at level $i . \Delta P_{i}^{\mathrm{M}}=P_{i+1}^{\mathrm{M}}-P_{i}^{\mathrm{M}}$ is the pressure difference between successive measurements. The superscript "M" stands for "measured". The $i$-th altitude level is simply

$z_{i}^{\mathrm{M}}=z_{0}+\sum_{k=0}^{i-1} \Delta z_{k}^{\mathrm{M}}$

Due to the measurement noise, the first differences of measured pressure, i.e. the series of the differences between successive measurements, are not always negative (as it should be during the ascent of the balloon) especially for high altitude levels, i.e. above $20 \mathrm{~km}$ altitude. The top panel of Fig. 1 shows the first differences of pressure measurements (cyan curve). It is observed that quantization effects are increasingly important with increasing altitudes. As a consequence, the calculated raw altitude, $z^{\mathrm{M}}$, from the measured pressure is not monotonically increasing during the ascent of the balloon: the altitude differences are quantized, the vertical steps growing with increasing altitudes (Fig. 1, lower panel), some differences being negative. Consequently, the raw altitudes $z^{\mathrm{M}}$ cannot be used for building the vertical profile.

A monotonic pressure profile is desirable for overcoming this difficulty. For that purpose, we estimate the pressure, $P^{\mathrm{A}}$, which, if quantized, would give the measured pressure, $P^{\mathrm{M}}$. The pressure $P^{\mathrm{A}}$ is evaluated through a least square cubic spline approximation (superscript "A" stands for "approximation"). The black curve of the upper panel of Fig. 1 shows the first differences $\Delta P^{\mathrm{A}}$ which are now negative for all levels. The altitude of measurements $z^{\mathrm{A}}$ is estimated from Eq. (1) with $P^{\mathrm{A}}$ instead of $P^{\mathrm{M}}$. In the lower panel of Fig. 1, the black curve shows the first differences of altitudes $\Delta z^{\mathrm{A}}$ estimated from $P^{\mathrm{A}}$ and $T^{\mathrm{M}}$. The vertical steps are found to range from 5 to $9 \mathrm{~m}$, due to the variations in the balloon ascent velocity.

For this specific flight (MUTSI-LR10) an average height interval of $7 \mathrm{~m}$ is found. The measured temperature $T^{\mathrm{M}}$ and approximated pressure $P^{\mathrm{A}}$ are resampled by interpolation with a regular vertical step of $7 \mathrm{~m}$. One can use either a 

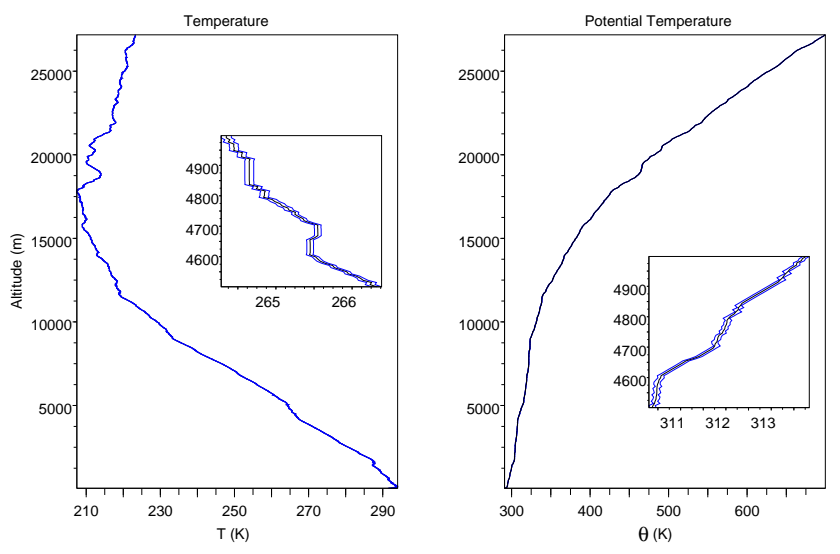

Fig. 2. Temperature profile (MUTSI-LR10 flight) (left). An expended view is shown for the 4500-5000 $\mathrm{m}$ altitude domain. Potential temperature profile (right).

linear interpolation or a cubic spline interpolation, the differences between these two methods being found to be negligible. The resampled temperature and pressure, $T^{\mathrm{R}}$ and $P^{\mathrm{R}}$, respectively are the data analyzed in the present work. Figure 2 (left panel) shows the temperature profile of flight LR10 of the MUTSI campaign. The curves show the temperature plus and minus two standard deviations of noise (only visible on the in the expended views in each panel). The method by which the instrumental noise is estimated will be described in Sect. 2.3.

The pressure, temperature and altitude are not independent quantities as they are related through the hydrostatic equilibrium relationship (Eq. 1). The knowledge of two of the three quantities fully determines the third one. For instance, the knowledge of $T^{\mathrm{R}}$ regularly sampled at step $\delta z$ allows to calculate the vertical profile of pressure for the same altitudes. The calculated pressure from $T_{i}^{\mathrm{R}}$ is almost the same as the resampled pressure $P_{i}^{\mathrm{R}}$ although $T$ and $P$ are independently measured: they differ by less than $0.02 \mathrm{~Pa}$ in the average, giving us strong confidence in the data processing method.

After re-sampling at a constant vertical step, the vertical resolution of LR temperature and pressure profiles is $\sim 6$ $7 \mathrm{~m}$, depending on the flight. The vertical resolution of the resampled HR profiles is $\sim 20 \mathrm{~cm}(10 \mathrm{~cm})$ for the SFT and MUTSI datasets, respectively.

The potential temperature $\theta$ is inferred from the resampled temperature and pressure, $T_{i}^{\mathrm{R}}$ and $P_{i}^{\mathrm{R}}$, by using the relation for ideal diatomic gas:

$\theta_{i}=T_{i}^{\mathrm{R}}\left(\frac{1000}{P_{i}^{\mathrm{R}}}\right)^{2 / 7}$

where $T$ is the temperature $(\mathrm{K})$, and $P$ the pressure $(\mathrm{hPa})$. The right panel of Fig. (2) shows the potential temperature plus and minus two standard deviations of noise. Several thick nearly neutralized layers can be seen in the troposphere,
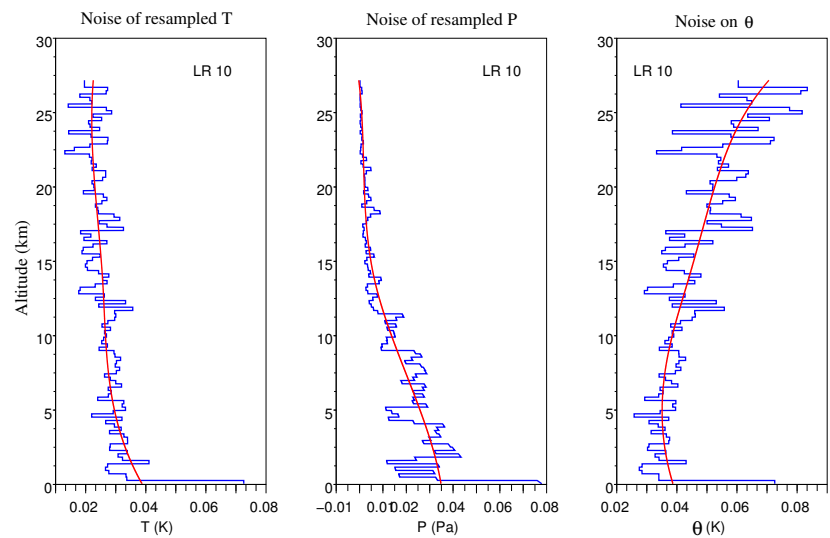

Fig. 3. Vertical profiles of the estimated level of noise for the MUTSI-LR10 flight of the temperature (left), the pressure (middle), the potential temperature (right). The noise level on potential temperature is inferred from the estimated noise on temperature and pressure. The blue curves show the estimates from data segments, the red curves showing smoothed profiles of these noise estimates obtained by spline approximations (see text).

around $4 \mathrm{~km}$ altitude and above $10 \mathrm{~km}$ altitude as well as in the stratosphere, below the altitude of $20 \mathrm{~km}$ altitude.

\subsection{Estimation of instrumental noise}

A key point of the selection method (presented in the next section) is to estimate the noise on potential temperature resulting from instrumental noise on $T$ and $P$. The noise standard deviations for the LR and HR data are experimentally estimated from both the temperature and pressure resampled profiles. After comparing several methods based on spectral or on wavelet analyses for some of them, we finally retained a simple-to-use method based on the estimate of the variance of the first differences of the data $(T$ or $\ln P)$. One important outcome of these tests is that the instrumental noise on temperature and pressure depends on altitude for both the LR and HR profiles. Therefore, we must estimate a vertical profile of the instrumental noise level. The method used for estimating the noise variance was made as follows:

1. Splitting the entire profile in segments of about $200 \mathrm{~m}$ in length (i.e. 32 or 2000 points according to the vertical resolution).

2. Removing a linear trend for each segment.

3. Calculating the variance of the first differences. This variance is an estimate of twice the noise variance.

4. Smoothing the resulting noise profile.

Figure 3 shows the estimated level of instrumental noise for the temperature and pressure measurements for flight MUTSI-LR10. The stairs step curve shows the noise estimates for each data segment, the smoothed curve displaying 
the noise level profile after smoothing (using a least square spline approximation). For the pressure measurements, the noise is estimated from the logarithm of the measurements in order to apply a linear detrending (step 2).

For the twenty LR flights of the SFT and MUTSI campaigns, the averaged noise standard deviation of the resampled temperature profile is found to be $27 \mathrm{mK}$. For the six HR flights, the noise standard deviation of temperature data is found to be $4 \mathrm{mK}$ in the average. The noise level of the LR temperature measurements is observed to decrease slightly with altitude (Fig. 3). The noise standard deviation of the resampled pressure profile is found to be negligible: $3 \times 10^{-2} \mathrm{~Pa}$ in the average. Consequently, the noise on the potential temperature essentially depends on the instrumental noise of temperature measurements. From Eq. (3), the standard deviation of noise of potential temperature, $\sigma_{\theta}$ is estimated by

$\sigma_{\theta}=\theta \sqrt{\left(\frac{\sigma_{T}}{T}\right)^{2}+\left(\frac{2}{7} \frac{\sigma_{P}}{P}\right)^{2}} \approx \theta \frac{\sigma_{T}}{T}=\left(\frac{1000}{P}\right)^{2 / 7} \sigma_{T}(4)$

where $\sigma_{T}\left(\sigma_{P}\right)$ are the noise standard deviation of temperature and pressure respectively. The noise level of potential temperature grows with altitude, due to the pressure term of Eq. (4): it increases from $\sim 35$ to $\sim 70 \mathrm{mK}(2.6$ to $3.5 \mathrm{mK}$ ) for LR (respectively HR) profiles for heights ranging from the ground level up to $27 \mathrm{~km}$.

\section{Analysis method of the potential temperature profiles}

\subsection{Selection method of overturns}

In order to evaluate the filtering effects due to both the vertical resolution and the level of instrumental noise, we analyzed the profiles by using an identical selection criterion. For discriminating overturns from inversions induced by noise, we used a hypothesis test on the range of the data. The method is described in detail by Wilson et al. (2010). Here, we shortly describe the three important steps of the method.

1. Determination of the optimal vertical resolution. We first estimate the vertical resolution that should be used by taking account for the mean stratification and the level of the instrumental noise. Such a resolution can be inferred from the bulk trend-to-noise ratio (tnr), $\bar{\zeta}$ of the profile. The bulk tnr, $\bar{\zeta}$, is a measure of the average of first differences of the data (potential temperature) $\bar{\tau}$, scaled by the noise $\sigma_{\theta}$ :

$$
\bar{\zeta}=\frac{\bar{\tau}}{\sigma_{\theta}}
$$

with

$$
\bar{\tau}=\overline{\theta_{i+1}-\theta_{i}}=\frac{1}{n-1}\left(\theta_{n}-\theta_{1}\right)
$$

where $\theta_{i}$ is the potential temperature measured at altitude $z_{i}(1 \leq i \leq n)$. The average of first differences $\tau$ depends on both the stability of the stratification and the vertical resolution since

$\bar{\tau}=\frac{\overline{\partial \theta}}{\partial z} \delta z$

where $z$ is the vertical coordinate and $\delta z$ the (constant) vertical step. The bulk tnr provides an indication on the minimum size for the overturns to be reliably detected (Wilson et al., 2010). The smaller $\bar{\zeta}$ be, the larger the data sample must be in order to be distinguishable from a pure noise sample. If $\bar{\zeta}$ is too small (smaller than 1 typically), a preliminary denoising procedure is required. The denoising procedure is based on both a filtering and an undersampling of the data. It aims at increasing the tnr while preserving the independence of the noise of each data bin. The tnr of the filtered and undersampled data increases by a factor $m^{3 / 2}$ where $m$ is the undersampling factor (i.e. the number of bins of the running filter).

2. Detection of the inversions. The potential temperature profile (denoised if needed) is sorted. The profile of Thorpe displacements can then be estimated. The (artificial and real) inversions are found from the cumulative sum of the Thorpe displacements since the sum of Thorpe displacements is null within an inversion.

3. Selection of the overturns. An inversion is identified as an overturn if the range of the potential temperature data belonging to the inversion exceeds a large prescribed percentile of the range of a noise sample of same length, with standard deviation equal to that of the instrumental noise. For practical purpose, we tabulated the moments of the range, as well as various percentiles, for normally distributed random variables as a function of the sample size (ftp.aero.jussieu.fr/pub/os/WN.txt).

The vertical profile of $\operatorname{tnr} \zeta_{\text {LR }}$ for the MUTSI-LR10 flight is shown in Fig. 4a. The bulk tnr $\bar{\zeta}_{\mathrm{LR}}$ is $\sim 0.9$ (dashed line), the local values showing minima smaller than $10^{-1}$ around $11 \mathrm{~km}$ altitude. For such a bulk tnr, one cannot expect to identify overturns smaller than $n \sim 60$ bins, i.e. $\sim 420 \mathrm{~m}$ (see Fig. 5 of Wilson et al., 2010). In order to improve the detection threshold, a denoising procedure is required. For the region with tnr of 0.1 , an undersampling $m=2$ allows to reach a local tnr of $2 \sqrt{2} \times 10^{-1} \approx 0.3$, allowing to detect overturns of 20 bins, i.e. $20 \times 14=280 \mathrm{~m}$.

\subsection{Overturns selection}

The average tnr being less than 1 for both LR and HR profiles in the troposphere, a denoising procedure is applied on the two datasets. The undersampling factor is 2 for the LR data, and is 5 (11) for the HR data of the SFT and MUTSI flights, 

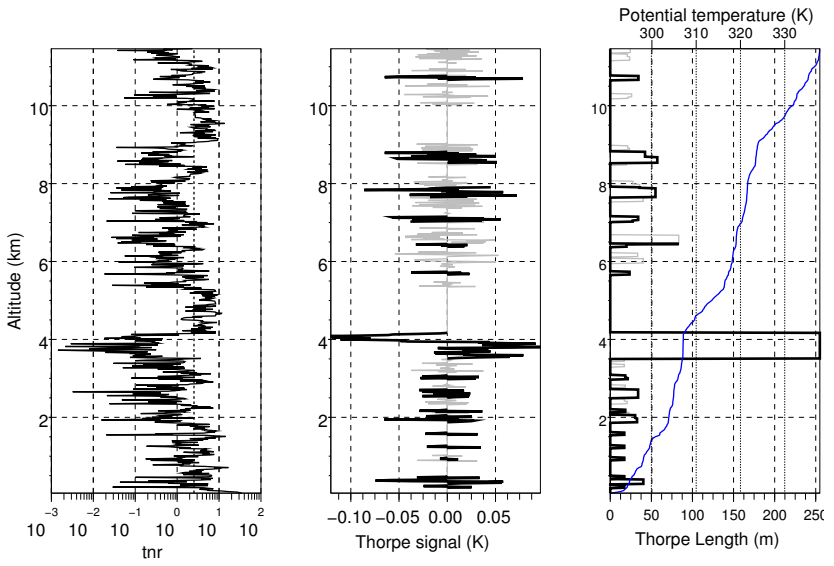

Fig. 4. MUTSI-LR10 flight: trend-to-noise ratio (left); Thorpe signal (middle); Thorpe lengths for the detected inversions (right). The selected overturns are highlighted.

respectively. The bulk tnr reaches $2-3$ for both datasets. Consequently, the vertical resolution is $\sim 12 \mathrm{~m}$ for the LR profiles, and is $1 \mathrm{~m}$ (SFT) or $1.1 \mathrm{~m}$ (MUTSI) for the HR profiles.

The tnr values of both LR and HR data are substantially larger in the stratosphere than in the troposphere due to the higher stability of the stratosphere. Consequently, the undersampling factor required to get a tnr of $\sim 2-3$ is smaller than in the troposphere, allowing to reach better vertical resolutions. The vertical resolution of the LR data is $\sim 6 \mathrm{~m}$ (no undersampling required) whereas the vertical resolution of the HR data is $\sim 0.5 \mathrm{~m}$.

In order to evaluate the impact of the selection method (including the denoising procedure) we compare the results of Thorpe analyses without and with the selection procedure. Figure 5 shows histograms of the number of occurrences of inversions (blue-filled) and overturns (red-transparent) as a function of their sizes for all the LR profiles. The distribution of the detected inversions (blue-filled histogram) results from the original potential temperature profiles (vertical resolution $\sim 6 \mathrm{~m}$ ), without applying any denoising and selection procedure. The distribution of the selected overturns for the same LR data (red-transparent histogram) results from the denoised (undersampled and filtered) profiles at a vertical resolution of $\sim 12 \mathrm{~m}$. The selection procedure shows that only $11 \%$ of the detected inversions can be regarded as overturns. Consequently, due to both the noise level of RS data and the average stability of the troposphere, most of the detected inversions $(91 \%)$ are only due to instrumental noise! It is therefore necessary to apply a selection procedure in order to identify real overturns, at least in the troposphere.

\section{Size distribution of the detected overturns}

We analyzed the LR and HR profiles by using the same selection method for detecting overturns. As mentioned in the

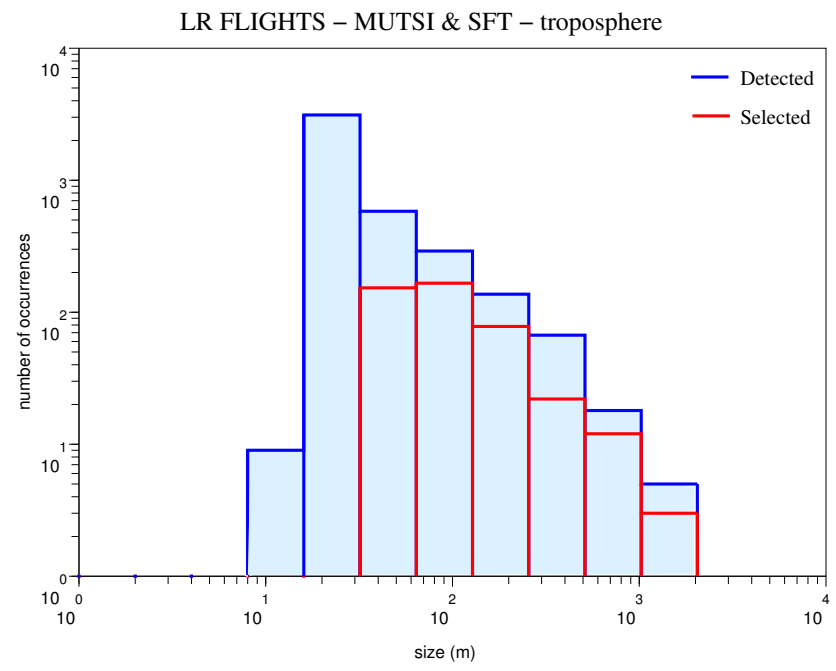

Fig. 5. Number of occurrences of the size of the detected inversions for all the LR profiles (blue-filled). Number of occurrences of the size of the selected overturns after applying a denoising and a selection procedure (red).

previous section, the bulk $\operatorname{tnr} \bar{\zeta}$ of all profiles ranges from 2 to 3 after applying the denoising procedure. We present in this section the empirical probability density functions (pdf), i.e. the relative frequencies, of the sizes of the selected overturns from both LR and HR datasets in the troposphere and in the stratosphere.

\subsection{Results for tropospheric data}

Figure 6 shows the number of occurrences of Thorpe displacements (in absolute value) as a function of their sizes for the six HR profiles. The two histograms result from all displacements found within the selected overturns in the troposphere. The corresponding histograms from the HR and LR profiles are shown in green-filled and red-transparent shadings, respectively. Due to the large dynamic of the observed values, the histograms are shown in a log-log scale. The widths of the intervals (abscissa) being constant on a log scale, they increase as a power law. We simply used a dyadic scale, the maximum value of each interval being equal to twice the minimum value (i.e. $1-2 \mathrm{~m}, 2-4 \mathrm{~m}$, and so on.). The number of occurrences is not directly comparable because the vertical step of LR profile is in the present case about 12 times larger than that of HR profiles ( $12 \mathrm{~m}$ vs. $1 \mathrm{~m})$. The considered displacements range from $1 \mathrm{~m}$ to $\sim 500 \mathrm{~m}$ and from 12 to $\sim 1000 \mathrm{~m}$ for the HR and LR profiles, respectively. As expected, the smallest displacements correspond to the vertical resolution, $\sim 1 \mathrm{~m}$ (within the $1-2 \mathrm{~m}$ interval) for HR data and $\sim 10 \mathrm{~m}$ (within the $8-16 \mathrm{~m}$ interval) for LR data.

On the other hand, about 20 displacements observed from LR profiles are larger than the largest displacement obtained from HR profiles. Such larger displacements in the LR 
Table 1. Number of occurrences and cumulative frequencies (brackets) of the size of selected overturns in the troposphere, for the MUTSI and SFT campaigns (6 HR and 20 LR profiles).

\begin{tabular}{lccccccccc}
\hline Sizes (m) & $2-4$ & $4-8$ & $8-16$ & $16-32$ & $32-64$ & $64-128$ & $128-256$ & $256-512$ & $512-1024$ \\
\hline HR & 245 & 1307 & 668 & 280 & 131 & 38 & 14 & 5 & 3 \\
& $(0.09)$ & $(0.58)$ & $(0.82)$ & $(0.93)$ & $(0.98)$ & $(0.992)$ & $(0.997)$ & $(0.999)$ & $(1)$ \\
\hline LR & 0 & 0 & 0 & 0 & 43 & 48 & 19 & 4 & 4 \\
(HR flights) & $(0)$ & $(0)$ & $(0)$ & $(0)$ & $(0.36)$ & $(0.76)$ & $(0.92)$ & $(0.95)$ & $(0.98)$ \\
\hline LR & 0 & 0 & 0 & 0 & 153 & 166 & 78 & 22 & 12 \\
(all flights) & $(0)$ & $(0)$ & $(0)$ & $(0)$ & $(0.35)$ & $(0.76)$ & $(0.92)$ & $(0.97)$ & $(0.99)$ \\
\hline
\end{tabular}

Thorpe Displacements - HR FLIGHTS - MUTSI \& SFT - Troposphere

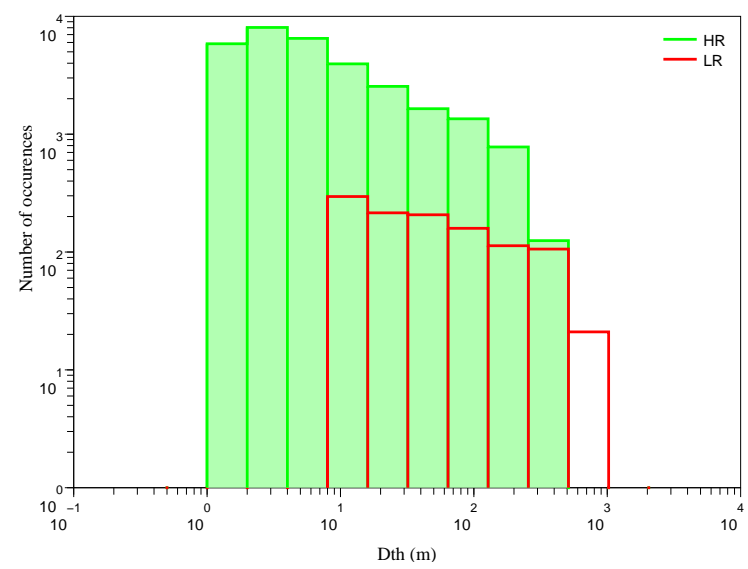

Fig. 6. Empirical distribution of Thorpe displacements within the selected overturns in the troposphere for the six HR flights of the MUTSI and SFT campaigns. The two histograms show the distributions obtained from HR profiles (green-filled) and LR profiles (red-transparent).

profile are likely the consequence of the poorer vertical resolution. Indeed, the LR profiles do not always allow to identify distinct overturns within weakly stratified regions. Such regions may appear as a single overturn in the LR profile, whereas the HR profile reveals several distinct overturns. Several examples of such merged overturns can be seen in the paper by Wilson et al. (2010). The largest LR displacements likely belong to these merged overturns. This effect is clearly a major source of bias when estimating Thorpe lengths and turbulence parameters from LR profiles in weakly stable regions.

The two histograms of Fig. (6) are proportional to the empirical pdf of Thorpe displacements. They reveal a tendency for the probability densities of displacements to decrease with increasing sizes. The pdf from LR measurements appears similar in shape but shifted by about $10 \mathrm{~m}$ toward larger scales.

Next, we consider the size of the selected overturns. The empirical pdf's of the overturn sizes for both LR and HR profiles are shown in the left panel of Fig. 7. The right panel
HR FLIGHTS - MUTSI \& SFT - Troposphere
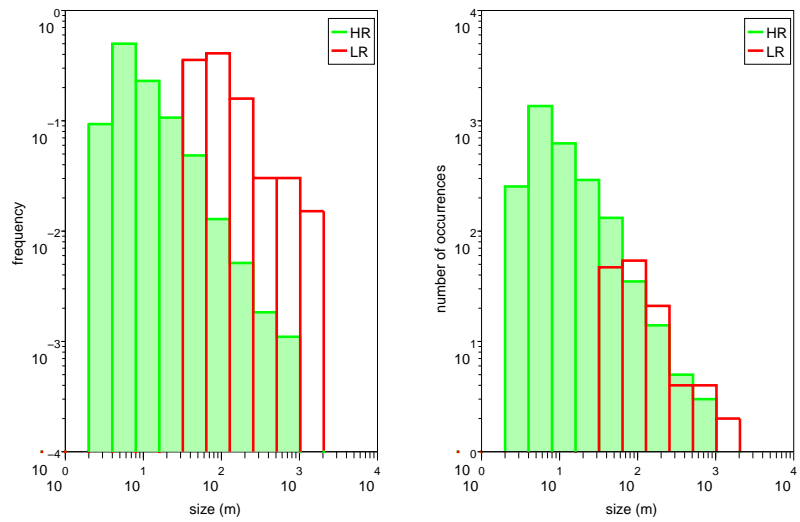

Fig. 7. Empirical pdf of the size of overturns for the six HR flights of the MUTSI and SFT campaigns (left). Number of occurrences of the size of overturns for the same six flights (right).

shows the occurrence number of overturns according to their size. The empirical pdf's are of course proportional to the number of occurrences. However, the plot of the occurrence numbers (right panel) allows a direct comparison of the numbers of events independently detected from HR and LR data during the six HR/LR flights. The size of the shallowest overturns from the HR data lies between 4 and $8 \mathrm{~m}$ whereas the shallowest overturns from LR data are within the 32-64 m interval. It should be noted that the inner scale of turbulence, which ranges from $\sim 0.1$ to $10 \mathrm{~cm}$ in the free troposphere and lower stratosphere (Eaton and Nastrom, 1998), is much smaller than the smallest scale detectable from the HR profiles. Consequently, the decreasing number of occurrences for the shallowest overturns in the HR profile is a cutoff effect similar to the one observed at much larger size for the LR profiles. The relative frequencies and the cumulative distributions of the overturn sizes are detailed in Table 1 . It is found that $93 \%$ of the overturns identified from the HR profiles are smaller than $32 \mathrm{~m}$, i.e. smaller than the shallowest overturns detected from the LR profiles. These results demonstrate that it is only possible to identify overturns which size belongs to the tail of the pdf from the LR 
Table 2. Number of occurrences and cumulative frequencies (brackets) of the size of selected overturns in the stratosphere, for the MUTSI and SFT campaigns (6 HR and 20 LR profiles).

\begin{tabular}{lccccccccc}
\hline Sizes (m) & $2-4$ & $4-8$ & $8-16$ & $16-32$ & $32-64$ & $64-128$ & $128-256$ & $256-512$ & $512-1024$ \\
\hline HR & 1960 & 1517 & 583 & 188 & 36 & 8 & 0 & 0 & 0 \\
& $(0.46)$ & $(0.81)$ & $(0.95)$ & $(0.990)$ & $(0.998)$ & $(1)$ & $(1)$ & $(1)$ & $(1)$ \\
\hline LR & 0 & 0 & 0 & 33 & 39 & 9 & 4 & 0 & 0 \\
(HR flights) & $(0)$ & $(0)$ & $(0)$ & $(0.39)$ & $(0.85)$ & $(0.95)$ & $(1)$ & $(1)$ & $(1)$ \\
\hline LR & 0 & 0 & 0 & 419 & 115 & 50 & 16 & 11 & 1 \\
(all flights) & $(0)$ & $(0)$ & $(0)$ & $(0.68)$ & $(0.87)$ & $(0.95)$ & $(0.98)$ & $(0.998)$ & $(1)$ \\
\hline
\end{tabular}

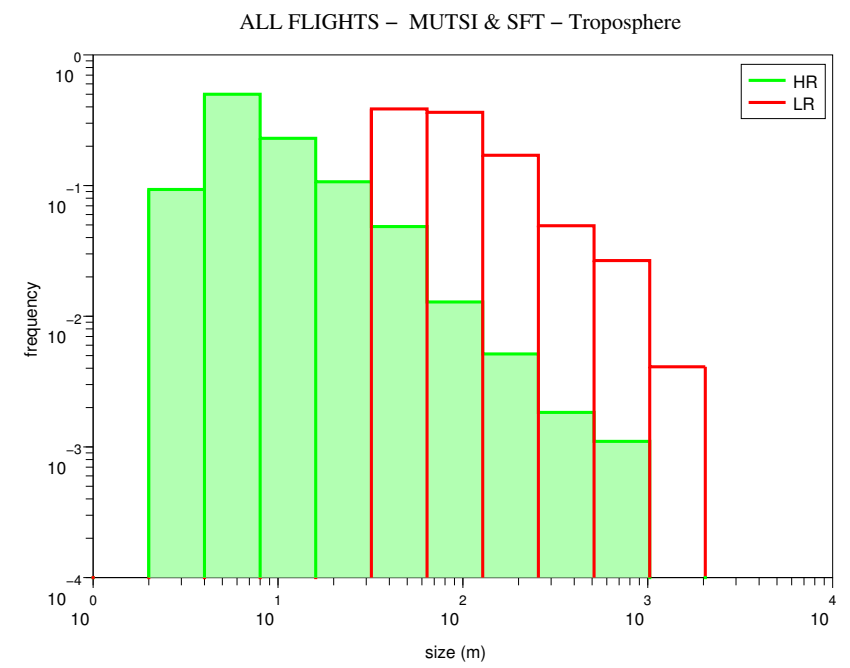

Fig. 8. Empirical pdf of of the size of the overturns for all the flights of the MUTSI and SFT campaigns (6 HR and 20 LR flights).

profiles in the troposphere. The selected overturns from the LR profiles represent $\sim 7 \%$ of the overturns found from HR profiles. On the other hand, the number of selected overturns from HR and LR profiles compares quite well, except for the smallest overturns in the LR profiles (the number of overturns in the HR profiles is about 3 times the number of overturns in the LR profiles for the $32-64 \mathrm{~m}$ interval). Although a large number of small overturns is missed, this result suggests that the largest overturns are correctly identified from the LR profiles.

Figure 8 shows the empirical pdf of the sizes of overturns for all the flights, i.e. the six HR and twenty LR profiles of the MUTSI and SFT campaigns, in the troposphere. Again, the smallest overturns of the LR profiles are within the 32$64 \mathrm{~m}$ interval. Also, the shape of the two probability densities compare quite well, as both densities decrease with a comparable rate. This observation suggests that the tail of the distribution in size of the overturns is correctly sampled from the LR profiles (except maybe for the extreme values, i.e. for the first and last intervals).

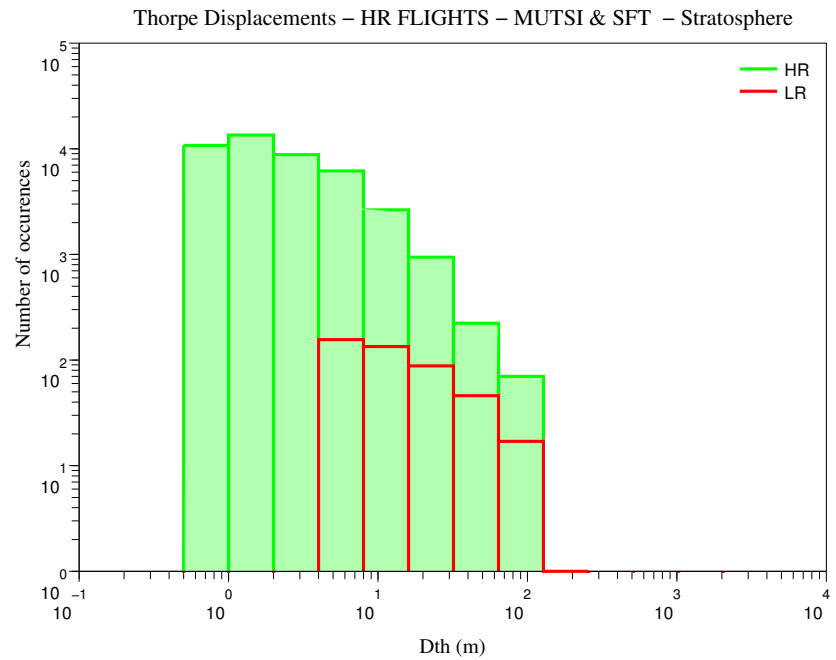

Fig. 9. Same as Fig. 6, but for the stratosphere.

\subsection{Results for stratospheric data}

The same selection method was applied to the HR and LR profiles collected in the stratosphere. Figure 9 shows the distribution of the number of occurrences of Thorpe displacements for the selected overturns (HR and LR) in the stratosphere for the six HR profiles. Here, the Thorpe displacements range from 0.5 to $\sim 50 \mathrm{~m}$ for the HR profiles, and from 6 to $\sim 200 \mathrm{~m}$ for the LR profiles. Again, the few larger displacements from LR data (less than 20 occurrences) are likely due to the much lower resolution of LR profiles, leading to a poor definition of the overturns within weakly stable regions.

The left panel of Fig. 10 shows the empirical pdf of the sizes of overturns in the stratosphere for the six HR profiles. From the HR profiles, the size of overturns now ranges from 2 to $\sim 100 \mathrm{~m}$. The relative frequencies of overturn size are found to sharply decrease with increasing size. From the LR profiles, the overturn size ranges from $\sim 25 \mathrm{~m}(16-32 \mathrm{~m})$ to $\sim 200 \mathrm{~m}(128-256 \mathrm{~m})$. Table 2 gives the cumulative frequencies of the overturn size. It is observed that $96 \%$ of the 
HR FLIGHTS - MUTSI \& SFT - Stratosphere

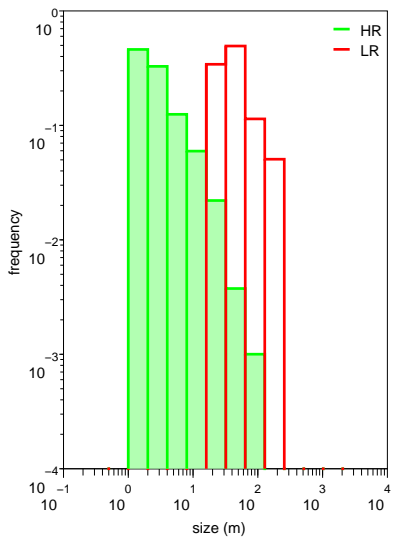

Fig. 10. Same as Fig. 7, but for the stratosphere.

selected overturns from the HR profiles have a size smaller than $16 \mathrm{~m}$ (Table 2). The right panel of Fig. 10 shows the number of occurrences of the overturn size for these six HR profiles. A striking feature is the small number of selected overturns from the six LR profiles: 85 events, i.e. about fourteen for each profile for all the sounded stratosphere (roughly from 10 to $25 \mathrm{~km}$ ). As in the troposphere, the number of occurrences of selected overturns according to their sizes is very similar for the LR and HR profiles (except for the smallest class, i.e. $16-32 \mathrm{~m}$, for the LR profiles). One can again concludes that, within the stratosphere, the overturns exceeding $\sim 25 \mathrm{~m}$ in size are correctly sampled from the LR profiles. But such overturns are rather rare in the stratosphere since they only represent about $4 \%$ of all the overturns detected from the HR profiles.

Figure 11 shows the empirical pdf of the overturn size in the stratosphere for all the (six HR and twenty LR) profiles. The sizes of overturns from the LR profiles now range from $\sim 25$ to $512-1024 \mathrm{~m}$. Both pdf's are sharply decreasing with increasing scales. The shape of the two distributions is very similar, giving extra credence to the detection of the larger overturns from the LR profiles. Again, the scales for which LR overturns are detected and selected represent only $4 \%$ of the HR overturns since the selection procedure does not allow to retain overturns smaller than $16 \mathrm{~m}$ from LR data.

\section{Conclusions}

We have analyzed a dataset of balloon soundings obtained during two field campaigns. The dataset includes six HR profiles $(10-20 \mathrm{~cm})$ and twenty LR profiles $(6-7 \mathrm{~m})$. The HR and LR profiles were obtained simultaneously, the sensors being on the same gondola. The LR profiles result from raw data collected from standard meteorological radiosondes vertically resampled at a constant step (6 or $7 \mathrm{~m}$ ).

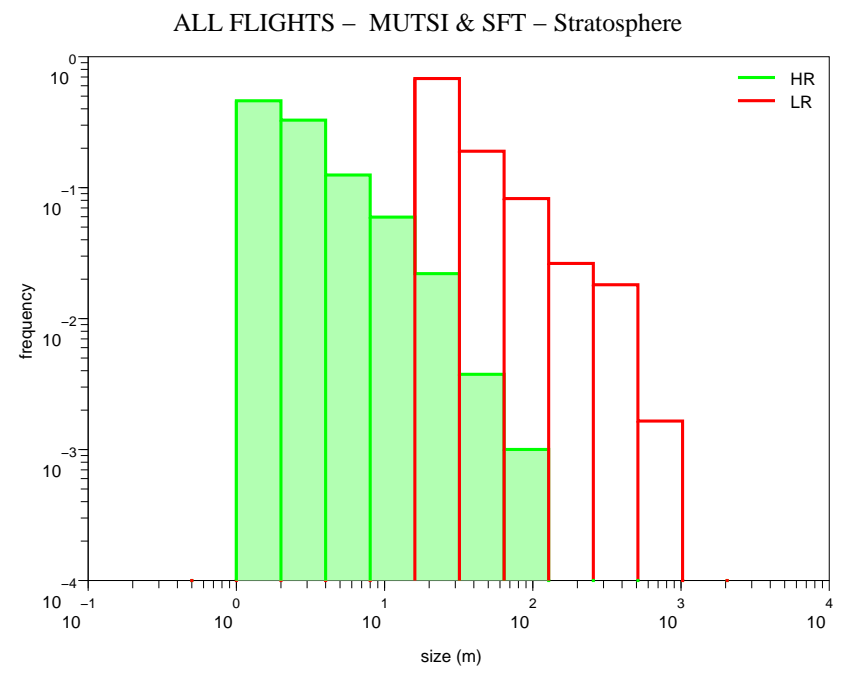

Fig. 11. Same as Fig. 8, but for the stratosphere.

After applying a denoising procedure, we performed a Thorpe analysis. We applied a selection method allowing to reject artificial inversions, i.e. inversions that cannot be distinguished from noise induced inversions. We can only retain $11.4 \%(25 \%)$ of the detected inversions as overturns from LR (respectively HR) profiles in the troposphere. In the stratosphere, the situation is worse as the noise on potential temperature increases whereas the sizes of the turbulent overturns diminishes. Only $7.9 \%$ (20.8\%) of the detected inversions are selected as overturns from the LR (respectively HR) profiles. Consequently, for detecting atmospheric turbulence from a Thorpe analysis, the impact of instrumental noise is a key issue which must be carefully considered in the troposphere as well as in the stratosphere.

A noticeable result of the present work, the interpretation of which is beyond the scope of this paper, is that the atmosphere is unstable for more than $56 \%$ in the troposphere, and more than $37 \%$ in the lower-stratosphere for vertical scales larger than one meter (from the HR profiles). The former value is in good agreement with the one reported by Cho et al (2003) who found that $54 \%$ of the troposphere is unstable when measured down to scale of $\sim 10 \mathrm{~m}$.

An important conclusion of the present work is that, despite the above mentioned limitations and difficulties, it is possible to detect overturns resulting from atmospheric turbulence from the raw data of standard meteorological radiosondes sampled at a rate of $0.7 \mathrm{~Hz}$. However, we showed that only the deepest overturns corresponding to the tail of the size pdf, can effectively be detected from standard meteorological radiosondes, in the troposphere as well as in the stratosphere. From our data set, the detectable overturns from RS in the troposphere correspond to the 7\% largest events that are observed from HR profiles. In the stratosphere, the detected overturns from RS correspond to about the $4 \%$ largest events. 
The latter conclusion opens interesting perspectives about the means of observing turbulence in the free atmosphere. Indeed, provided that raw data of radiosondes are available, and by applying a rigorous treatment for the instrumental noise, the huge data base of standard meteorological radiosoundings can be used in order to carry out a climatology of atmospheric turbulent events through the detection of overturns, at least for the most energetic events.

Acknowledgements. The authors wish to acknowledge CNES (Centre National d'Études Spatiales) for operating the balloons in Japan and the Research Institute for Sustainable Humanosphere (RISH) for their contribution to the MUTSI campaign.

Edited by: S. Buehler

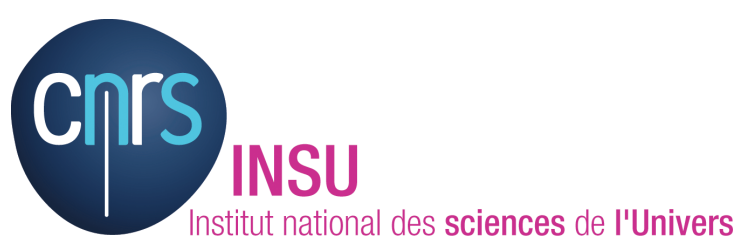

The publication of this article is financed by CNRS-INSU.

\section{References}

Alappattu, D. P. and Kunhikrishnan, P. K.: First observations of turbulence parameters in the troposphere over the Bay of Bengal and the Arabian Sea using radiosonde, J. Geophys. Res., 115, D06105, doi:10.1029/2009JD012916, 2010.

Alford, M. H. and Pinkel, R.: Observation of overturning in the thermocline: The context of ocean mixing, J. Phys. Oceanogr., 30, 805-832, 2000.

Balsley, B., Kantha, L., and Colgan, W.: On the use of Slow Ascent Meter-Scale (SAMS) Radiosondes for observing overturning events in the free atmosphere, J. Atmos. Ocean. Tech., 27, 766-775, 2010.

Clayson, C. A. and Kantha, L.: On Turbulence and Mixing in the Free Atmosphere Inferred from High-Resolution Soundings, J. Atmos. Ocean. Tech., 25, 833-852, 2008.
Cho, J. Y. N., Newell, R. E., Anderson, B. E., Barrick, J. D. W., and Thornhill, K. L.: Characterizations of tropospheric turbulence and stability layers from aircraft observations, J. Geophys. Res., 108(D20), 8784, doi:10.1029/2002JD002820, 2003.

Eaton, F. D. and Nastrom, G. D.: Preliminary estimates of the vertical profiles of inner and outer scales from White Sands Missile Range, New Mexico, VHF radar observation, Radio Sci., 33, 895-903, 1998.

Ferron, B., Mercier, H., Speer, K., Gargett, A., and Polzin, K.: Mixing in the Romanche fracture zone, J. Phys. Oceanogr., 28, 1929 1945, 1998.

Galbraith, P. S. and Kelley, D. E.: Identifying overturns in CDT profiles, J. Atmos. Ocean. Tech., 13, 688-702, 1996.

Gargett, A. E. and Garner, T.: Determining Thorpe Scales from Ship-Lowered CTD density profiles, J. Atmos. Ocean. Tech., 25, 1657-1670, 2008.

Gavrilov, N. M., Luce, H., Crochet, M., Dalaudier, F., and Fukao, S.: Turbulence parameter estimations from high-resolution balloon temperature measurements of the MUTSI-2000 campaign, Ann. Geophys., 23, 2401-2413, doi:10.5194/angeo-23-24012005, 2005.

Johnson, H. L. and Garrett, C.: Effect of noise on Thorpe Scales and run lengths, J. Phys. Oceanogr., 34, 2359-2372, 2004.

Luce, H., Fukao, S., Dalaudier, F., and Crochet, M.: Strong mixing events observed near the tropopause with the MU radar and hight-resolution Balloon techniques, J. Atmos. Sci., 59, 28852896, 2002.

Nath, D., Venkat Ratman, M., Patra, A. K., Krishna Murthy, B. V., and Bhaskar Rao, S. V.: Turbulence characteristics over tropical station Gadanki $\left(13.5^{\circ} \mathrm{N}, 79.2^{\circ} \mathrm{E}\right)$ estimated using highresolution GPS radiosonde data, J. Geophys. Res., 115, D07102, doi:10.1029/2009JD012347, 2010.

Thorpe, S. A.: Turbulence and mixing in a Scottish Lock, Philos. T. Roy. Soc. Lond. A, 286, 125-181, 1977.

Wilson, R.: Turbulent diffusivity in the free atmosphere inferred from MST radar measurements: a review, Ann. Geophys., 22, 3869-3887, doi:10.5194/angeo-22-3869-2004, 2004.

Wilson, R., Luce, H., Dalaudier, F., and Lefrère, J.: Turbulent Patch Identification in Potential Density/Temperature Profiles, J. Atmos. Ocean. Tech., 26, 977-993, 2010. 